\title{
Unsymmetrical cyanine dye via in vivo hitchhiking endogenous albumin affords high-performance NIR-II/photoacoustic imaging and photothermal therapy
}

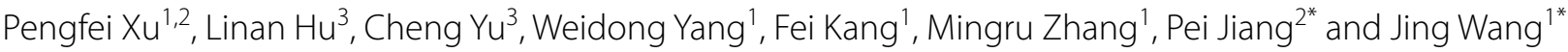

\begin{abstract}
Herein, an unprecedented synergistic strategy for the development of high-performance NIR-II fluorophore is proposed and validated. Based on an unsymmetrical cyanine dye design strategy, the NIR-II emissive dye NIC was successfully developed by replacing only one of the indoline donors of symmetrical cyanine dye ICG with a fully conjugated benz[c,d]indole donor. This minor structural change maximally maintains the high extinction coefficient advantage of cyanine dyes. NIC-ER with endogenous albumin-hitchhiking capability was constructed to further enhance its in vivo fluorescence brightness. In the presence of HSA (Human serum albumin), NIC-ER spontaneously resides in the albumin pocket, and a brilliant $~ 89$-fold increase in fluorescence was observed. Due to its high molar absorptivity and moderate quantum yield, NIC-ER in HSA exhibits bright NIR-II emission with high photostability and significant Stokes shift (>110 nm). Moreover, NIC-ER was successfully employed for tumor-targeted NIR-II/PA imaging and efficient photothermal tumor elimination. Overall, our strategy may open up a new avenue for designing and constructing high-performance NIR-II fluorophores.
\end{abstract}

\section{Introduction}

Recently, optical imaging in the "second near-infrared (NIR-II) window" (1000-1700 $\mathrm{nm}$ ) has been considered as an ideal imaging modality than traditional NIR-I (650$900 \mathrm{~nm}$ ) imaging [1-3]. So far, the most active materials used for NIR-II imaging and therapy are mainly nanoparticles (single-walled carbon nanotubes and lanthanide nanoparticles) and quantum dots [4-7]. In terms of clinical translation ability, small organic fluorophores offer a better alternative due to their compact molecular

\footnotetext{
*Correspondence: jiangpeicsu@sina.com; wangjing@fmmu.edu.cn 1 'Department of Nuclear Medicine, Xijing Hospital, Fourth Military Medical University, \#127 West Changle Road, Shanxi 710032 Xi'an, People's Republic of China

${ }^{2}$ Institute of Clinical Pharmacy and Pharmacology, Jining First People's Hospital, Jining Medical University, Jining 272000, People's Republic of China

Full list of author information is available at the end of the article
}

structure, minimal toxicity, and facile derivatization [8-16]. Interestingly, typical NIR I fluorophore ICG, one of the most famous cyanine dyes, can be used for NIRII imaging by exploiting the off-peak emission beyond $1000 \mathrm{~nm}[8,17-20]$. However, the off-peak emission of ICG maybe not be bright enough for deep tissue NIR-II imaging. To achieve longer emission, conjugated donor groups (benz $[\mathrm{c}, \mathrm{d}]$ indole and indolizine) have been introduced into the cyanine bridge to delocalize the frontier molecular orbitals further. For example, replacing the indoline donor of typical indoline cyanine dyes with a fully conjugated benz[c,d]indole donor results in a redshift emission of above $200 \mathrm{~nm}$ [21]. In another case, heptamethine cyanine dye FD-1080 uses two benz[c,d] indoles as terminal heterocycles affording maximal emission wavelengths over $1000 \mathrm{~nm}$, although this come at the expense of decreased molar extinction coefficient original author(s) and the source, provide a link to the Creative Commons licence, and indicate if changes were made. The images or other third party material in this article are included in the article's Creative Commons licence, unless indicated otherwise in a credit line to the material. If material is not included in the article's Creative Commons licence and your intended use is not permitted by statutory regulation or exceeds the permitted use, you will need to obtain permission directly from the copyright holder. To view a copy of this licence, visit http://creativecommons.org/licenses/by/4.0/. The Creative Commons Public Domain Dedication waiver (http://creativeco mmons.org/publicdomain/zero/1.0/) applies to the data made available in this article, unless otherwise stated in a credit line to the data. 
$\left(2.9 \times 10^{4} \mathrm{M}^{-1} \mathrm{~cm}^{-1}\right.$ vs. $2.3 \times 10^{5} \mathrm{M}^{-1} \mathrm{~cm}^{-1}$ of ICG) [22]. Although NIR-II fluorescence imaging has achieved encouraging results in tumor imaging and angiography, most of the reported organic NIR-II fluorophores suffer from low molar absorptivity, complicated synthesis steps, low aqueous solubility, or poor quantum yields [23-25]. There is still long way to go before NIR-II dyes are ready for clinical applications.

In the present work, we apply a novel design strategy to develop a nonsymmetrical NIR-II emitting cyanine dye, where only one of the indoline donors of the typical symmetrical cyanine dye ICG was replaced by a fully conjugated benz[c,d]indole donor. This minor structure change extending the $\pi$-conjugation of the dye allows its emission red-shift into the NIR-II region while maximally maintaining the advantage of the high extinction coefficient of the parent dye (ICG). According to energy-gap law, NIR-II emissive dyes are inherently low fluorescence quantum because the generation of NIR-II emission photons requires low energy bandgap; thus, inducing non-radiative decay processes tend to dominate over radiative photon emission [26]. One method of reducing non-radiative deactivation is intercalation of organic dyes comprising large, hydrophobic $\pi$-systems into plasma proteins to restrict their conformational freedom $[27,28]$. As stated in previous report, $\mathrm{CH}-4 \mathrm{~T}$ exhibits a 110-fold fluorescence increase after forming dye-protein molecular complexes by incubation with FBS at 70 ${ }^{\circ} \mathrm{C}$ for $10 \mathrm{~min}$ [29]. Another promising method is to link NIR-II dyes with an albumin binder so that the conjugate can bind to circulating endogenous albumin in situ after intravenous injection. Evans blue (EB) is a non-toxic azo dye with high serum albumin binding affinity and excellent hydrophilicity [30, 31]. More recently, a maleimidemodified truncated Evans Blue (tEB) was developed as an "add-on" molecule for a variety of bioconjugation applications in diseases imaging and treatment [32, 33]. We hypothesized that the conjugation of NIR-II dye with tEB could afford the conjugate with endogenous albumin hitchhiking capability, which would increase its fluorescence brightness and extend its half-life in the blood [34-36]. Besides, slow release of the NIR-II emissive conjugate over time would allow continual uptake at the biological target [37, 38]. To the best of our knowledge, this designing strategy for high-performance NIR-II fluorophores has never been reported $[39,40]$.

Based on the above molecular design principle, we develop a novel unsymmetrical cyanine dye NIC. To impart NIC with albumin-binding and active-targeting abilities, an albumin-binding motif tEB (truncate Evans blue) and an active targeting ligand c(RGDfc) were introduced into the molecular structure of NIC (termed as NIC-ER). Compared to benchmark ICG, NIC exhibits red-shifted absorption and emission with dichromic properties. NIC-ER was easily loaded into the pocket of albumin in the presence of albumin, which induces a dominant emission at $1030 \mathrm{~nm}$ and a brilliant $~ 89$-fold increase in fluorescence brightness. The absolute quantum yield of NIC-ER in HSA (Human serum albumin, $25 \mathrm{mg} / \mathrm{mL}$ ) was measured to be $3.56 \%$, which is higher than most reported organic NIR-II dyes [45-47]. The NIC-ER in HSA exhibits bright NIR-II emission with high photostability and significant Stokes shift (>110 nm) due to the high molar absorptivity and moderate quantum yield. Then, noninvasive imaging of small blood vessels at the limb, cerebral, and abdomen region is achieved with a high signal-to-background ratio and deep penetration. Furthermore, NIC-ER has also been successfully applied for targeted tumor imaging (NIR-II fluorescence/ PA synergistic imaging) and efficient tumor elimination (photothermal therapy). In summary, this study offers a superior NIR-II cyanine dye with satisfying brightness, large Stokes-shift, and high photostability and opens up a new avenue for designing and constructing high-performance NIR-II fluorophores.

\section{Results and discussion}

As shown in Additional file 1: Scheme S1, NIC and NICER were easily synthesized. The synthesis began with the heterocyclic salts and $N$-[5-(phenylamino)-2,4-pentadienylidene] aniline monohydrochloride. This reaction proceeded well in $58 \%$ yield affording NIC. NIC-MLEB was prepared through the direct conjugation of the carboxylic acid group of NIC with the compound MLEB. Then, the free thiol group of $\mathrm{c}(\mathrm{RGDfc})$ was covalently attached to the maleimide motif of NIC-MLEB to produce the final product NIC-ER.

With NIC in hand, its spectroscopic properties were first studied in various solvents. Typical to cyanine dyes, NIC exhibits a maximum absorption peak with a blueshifted shoulder, even though the shoulder has a relatively enhanced contribution in the overall absorption spectrum. Figure $1 \mathrm{~b}$ shows that the solvents had a significant effect on the absorption curve shape of NIC. DMSO shows the closest ratio of high-energy to lower-energy features nearing equal heights. High-energy absorption is the dominant feature in THF and DCM, while low-energy absorption is dominant in $\mathrm{MeOH}$. The molar absorptivities of NIC differ from $1.3 \times 10^{5}$ to $2.0 \times 10^{5} \mathrm{M}^{-1} \mathrm{~cm}^{-1}$ depending on the choice of the solvent. Due to planar $\pi$-electron conjugation systems, typical cyanine dyes tend to self-aggregate in aqueous buffer solutions, forming two different structures ( $\mathrm{J}$-aggregations and $\mathrm{H}$-aggregations) depending on the angle of slippage $(\alpha)$ [41]. This phenomenon was also observed in this study. When NIC was directly added to PBS (high concentrations, $\geq$ 


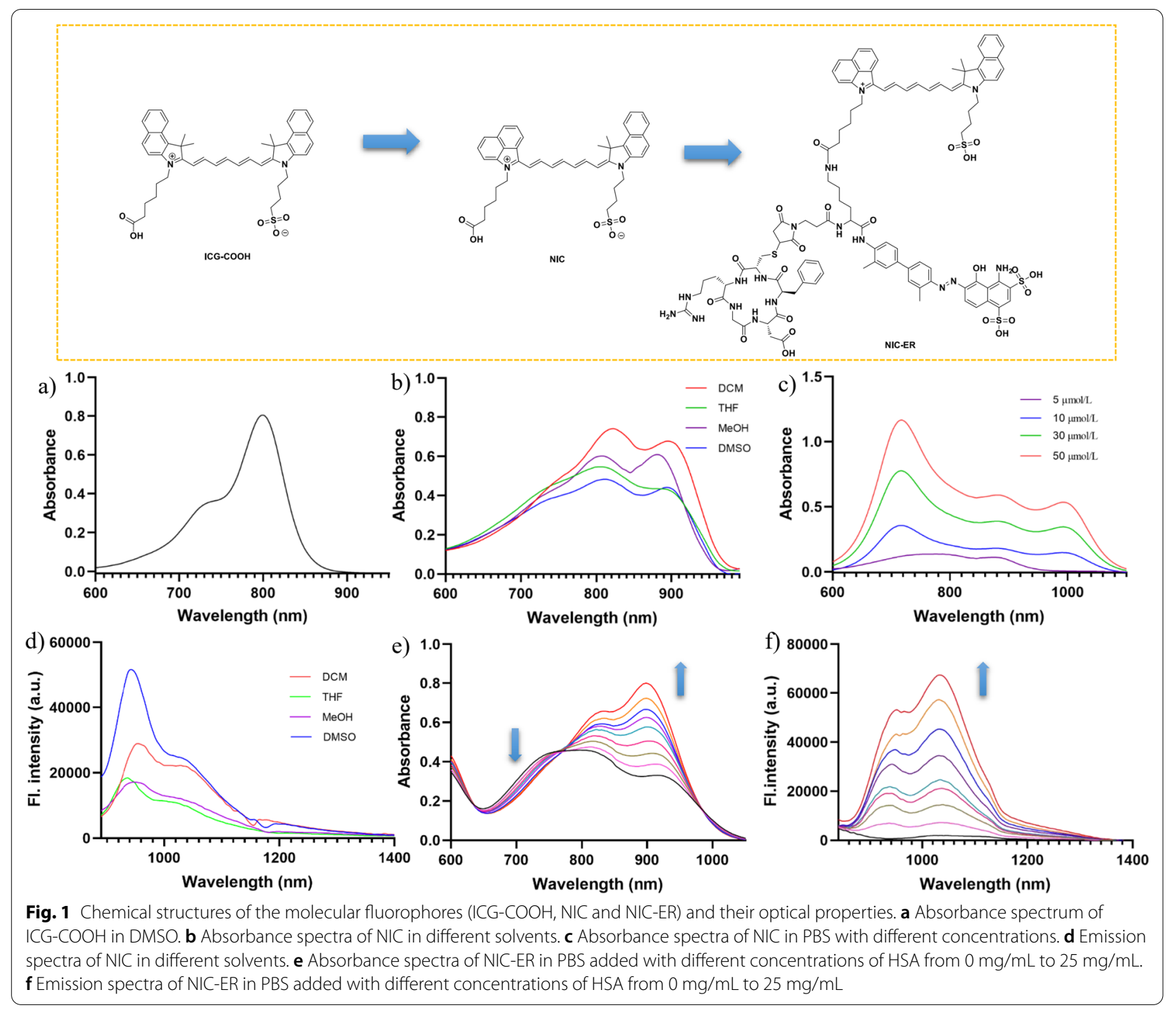

$\left.5 \times 10^{-6} \mathrm{M}\right)$, H-aggregates with an absorption band blueshifted to $700 \mathrm{~nm}$ and J-aggregates with absorption band red-shifted to $1000 \mathrm{~nm}$ were observed simultaneously (Fig. 1c).

Compared to the reference ICG-COOH with an absorption maximum at $800 \mathrm{~nm}$, there is approximately a $100 \mathrm{~nm}$ red-shift, which can be tentatively ascribed to the further conjugated electron-deficient system in the benz $[\mathrm{c}, \mathrm{d}]$ indole heterocycle. Density functional theory calculation was conducted (Fig. 2a, b) to examine the geometric and electronic properties of NIC and ICG-COOH. For ICG-COOH, the HOMO is uniformly distributed between the indoline nucleus ring and the polymethine chain systems, while the LUMO is mostly on the polymethine chain. The HOMO and LUMO are more extended to the conjugated benz[c,d]indole heterocycle in NIC, which would maximize the $\pi$-orbital overlap and oscillator strength. Furthermore, this decreases the HOMOLUMO gap, which is consistent with the experimentally observed bathochromic shift in absorption.

The emission curve shapes of NIC variation with solvents are shown in Fig. 1d. All test solvents show an emission peak at $950 \mathrm{~nm}$ and a shoulder at $1030 \mathrm{~nm}$, and the ratio of the two emissions varied with solvents. DMSO represents the highest ratio of high-energy to low-energy peak at about 2, while the remaining solvents all exhibit a ratio of 1.4 or less. No obvious emission peak was observed upon dissolution of NIC in PBS, which could be plausibly attributed to the strong excitonic interaction in the aggregates. In contrast to the high aggregation propensity of NIC, NIC-ER exhibited a dramatically decreased degree of aggregation at the same 


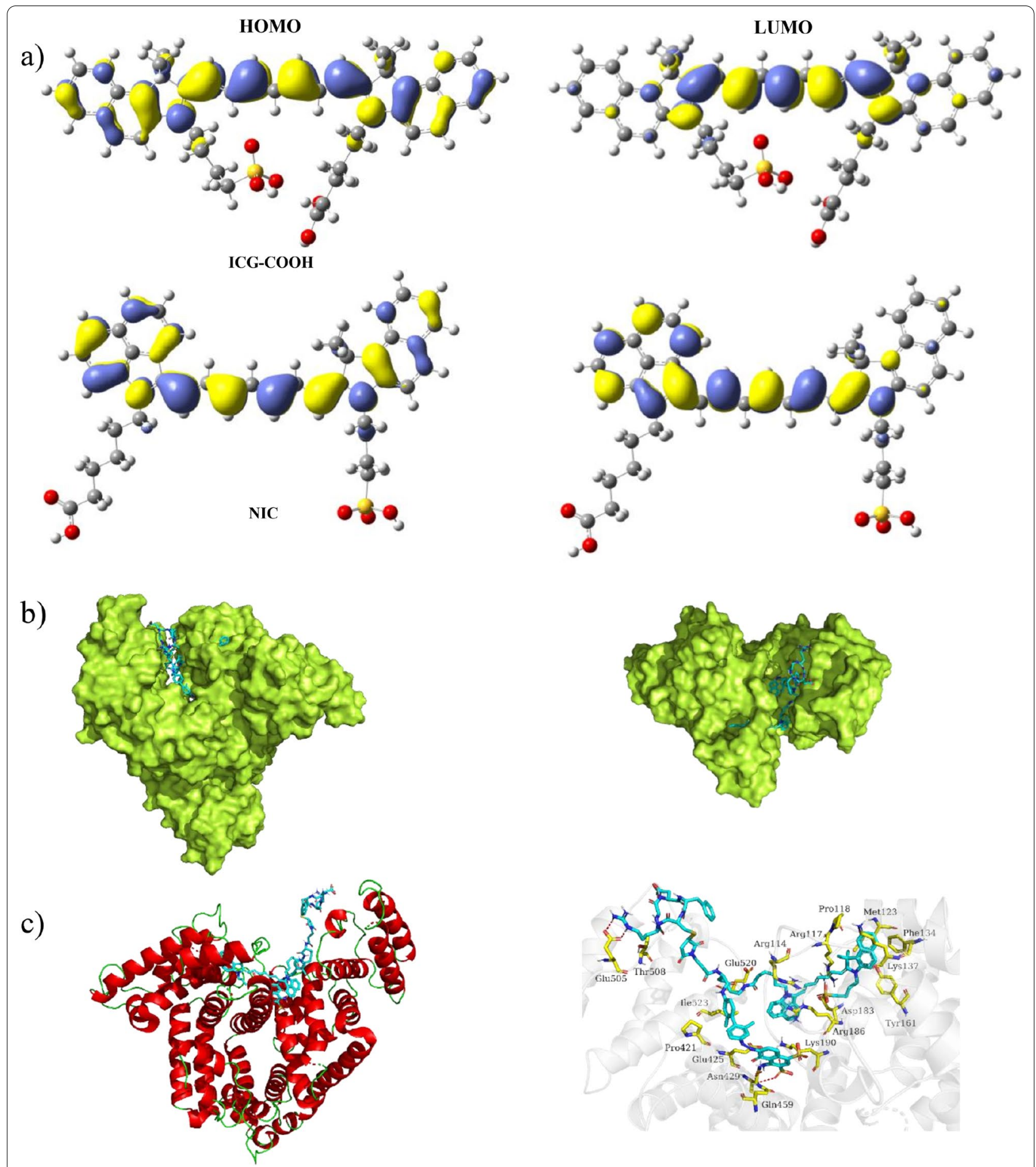

Fig. 2 a HOMO and LUMO wave functions in the geometrically optimized structures of ICG-COOH and NIC. b-c The possible interaction modeling between NIC-ER and albumin. b Docking modeling for the NIC-ER @albumin complex. c Details of binding residues and active pocket between NIC-ER and albumin 
concentration in PBS. After mixing free NIC-ER dye with HSA, the truncate Evans blue motif exerts its albuminbinding effect, and this binding model induces a drastic change of absorption curve shape. As the level of HSA content increased, the intensity of the absorption peak and shoulder both increased, but with different enhancement levels. Figure 1e shows that the absorption at 900 $\mathrm{nm}$ possesses more enhancement relative to shorter wavelength absorption $(800 \mathrm{~nm})$, and the $910 / 800 \mathrm{~nm}$ absorption ratio changed from 0.8 (PBS) to 1.3 (25 mg/ mL HSA). Furthermore, a similar enhancement trend was also observed in the emission data. Figure if shows that after mixing free NIC-ER dye with HSA, both the smaller emission peak at $950 \mathrm{~nm}$ and higher emission peak at $1030 \mathrm{~nm}$ enhanced, and a drastic $\sim 89$-fold increase in fluorescence was observed. With the addition of HSA, a longer emission peak at $1030 \mathrm{~nm}$ gradually increased the contribution to the overall fluorescence spectra. In the solution containing $25 \mathrm{mg} / \mathrm{mL} \mathrm{HSA}$, the $1030 / 950 \mathrm{~nm}$ emission ratio increased to 1.25 .

Even though it is well known that intercalation of dyes into the HSA pocket can lead to emission enhancement due to two events: disruption of aggregation and constrained geometry, which reduces non-radiative decay [33, 42]. However, this cannot explain why the emission peaks of NIC-ER (950 nm and $1030 \mathrm{~nm}$ ) have different enhancement levels in the presence of HSA. From the resonance perspective, unsymmetrical cyanine dyes are characterized by two nonidentical extreme resonance configurations with different energy, which may be responsible for dichromic absorption and emission [43]. Unlike the symmetrical ICG in which the positive charge is delocalized in the entire fluorophore, the structure of NIC raises a possibility that the positive charge could be preferentially localized on one of the heterocycle $\mathrm{N}$ atoms in the ground state. Undoubtedly, the more stable configuration is the one in which the nitrogen of the more basic nucleus is quaternary [44]. Thus, the relative contribution of these two resonance forms determines the ratio of the two peaks in dichromic absorption in dichromic emission. We performed docking modeling to investigate the positions and interaction between NIC-ER and albumin (Fig. 2c, d). The binding site of NIC-ER was identified as the center cleft of albumin, which is consistent with a previous study that the cleft on albumin is the preferred binding site of truncate Evans blue [38]. In the binding pocket, the positively charged benz[c,d]indole heterocycle could further be stabilized by the nearby amino acids through electronic coupling and inductive effects, inducing the dominant absorption and emission at a longer wavelength range.

Besides, the absolute quantum yield of NIC-ER in HSA $(25 \mathrm{mg} / \mathrm{mL}$ ) was measured to be $3.56 \%$, which is higher than most reported organic NIR-II dyes [45-47]. Importantly, NIC-ER has satisfied molecular brightness with great potential for efficient bioimaging. Photostability and biocompatibility of NIR-II dyes are also crucial for their biomedical applications. The cellular toxicity of NIC-ER was evaluated using a CCK-8 assay (Additional file 1: Figure S1). The cellular viability of healthy human cervical cancer cells (Hela) shows a survival rate of above $90 \%$ at the concentration of $0-50 \mu \mathrm{mol} / \mathrm{L}$, suggesting the low toxicity of NIC-ER. In vivo toxicity of NIC-ER was performed after intravenous injection at $5 \mathrm{mg}$ per $\mathrm{kg}$ mouse (10 times than the imaging doses). No significant changes were found in main organs (heart, liver, spleen, lung, and kidney) after 7 days post-injection (Additional file 1: Figures S7), suggesting low in vivo toxicity of NICER. Additional file 1: Figure S2 shows that NIC-ER in HSA exhibits better photostability than ICG-COOH and NIC-ER in PBS under continuous laser irradiation for $15 \mathrm{~min}$. The remarkably high photostability of NIC-ER makes it very attractive for use in long-term tracking or fluorescence-guided surgery, which requires long periods of sustained light exposure [48].

We injected NIC-ER intravenously into BALB/c nude mice at the dose of $0.5 \mathrm{mg} / \mathrm{Kg}$ to investigate the potential of NIC-ER as a novel NIR-II contrast agent for bioimaging. Excitation was achieved using a $915 \mathrm{~nm}$ diode laser at $100 \mathrm{~mW} \mathrm{~cm}{ }^{-2}$, and NIR-II fluorescence images were captured through a $1250 \mathrm{~nm}$ LP filter (exposure time, $100 \mathrm{~ms})$. After intravenous injection of NIC-ER, the hind limb vessels were clearly distinguished, revealing the high spatial resolution of NIR-II fluorescent imaging (Fig. 3a). Meanwhile, the fluorescence intensities of blood vessels and muscles in the hind limb were measured, and the signal-to-background ratio (SBR) was evaluated to be 3.2 (Additional file 1: Figure S5). Furthermore, the vessel resolved by NIR-II showed a maximum feature resolution (full width at half maximum, FWHM) of $0.34 \mathrm{~mm}$, which is consistent with the previously reported value [30]. Furthermore, the whole abdomen vessels, including the inferior epigastric, can be imaged (Fig. 3b). The above results inspired us to probe deep tissue. Compared with the skin surface vessels, cerebral vasculature is located much deeper $(\approx 1.3 \mathrm{~mm})$. We then further explored the imaging performance of NIC-ER for in vivo cerebral vasculature imaging through the scalp and the skull (cranial bone) without craniotomy. The large and detailed vessels were clearly distinguished (Fig. 3c) after intravenous injection of NIC-ER. Next, we explored the application of NIC-ER to lymphatic imaging. Fluorescence images were obtained after intradermal injection of NIC-ER at the rear paw of nude mice. Figure 3d shows that the popliteal lymph nodes and the lymphatic ducts were visualized very clearly. These results show that NIC-ER is qualified 

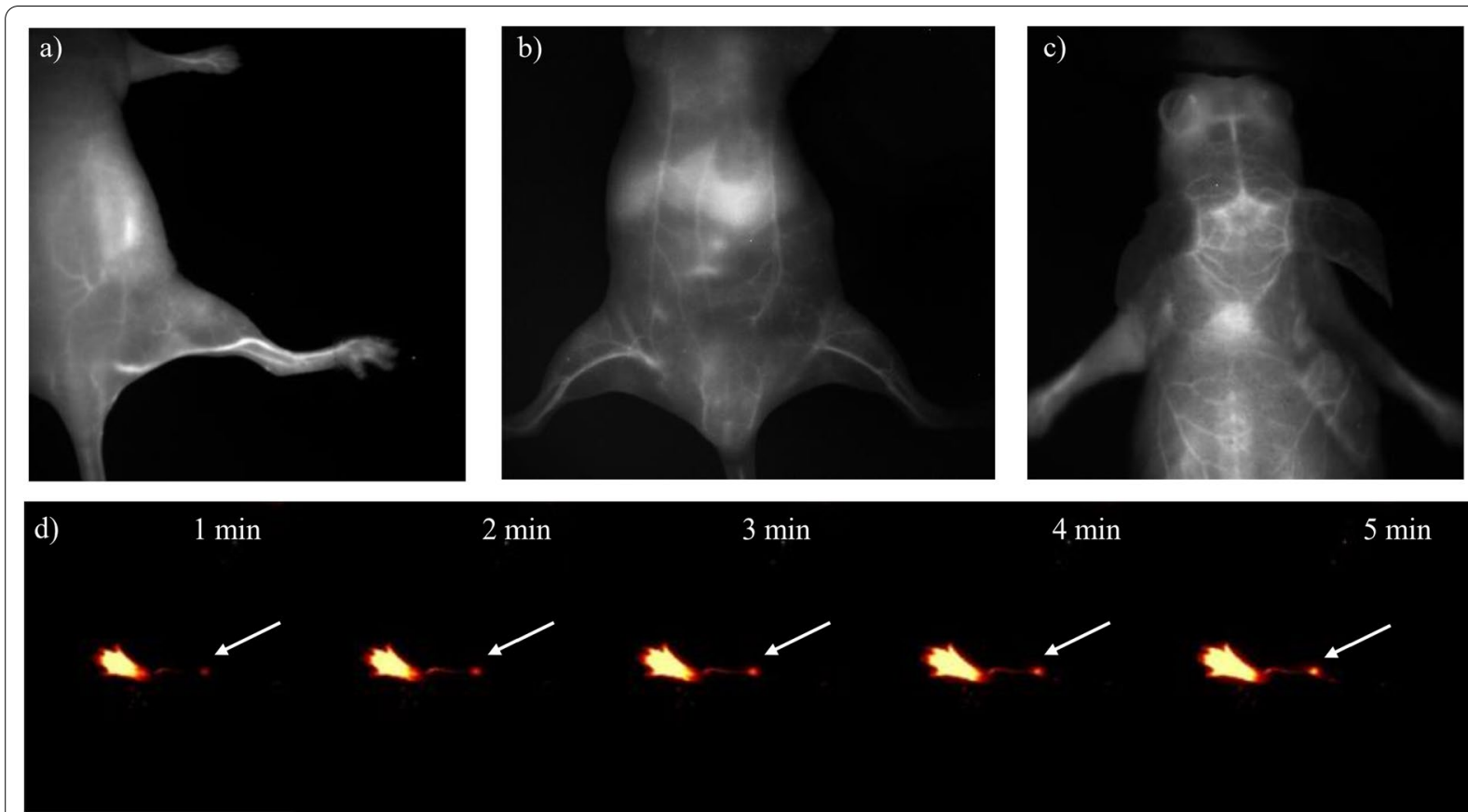

$4 \min$

$5 \mathrm{~min}$

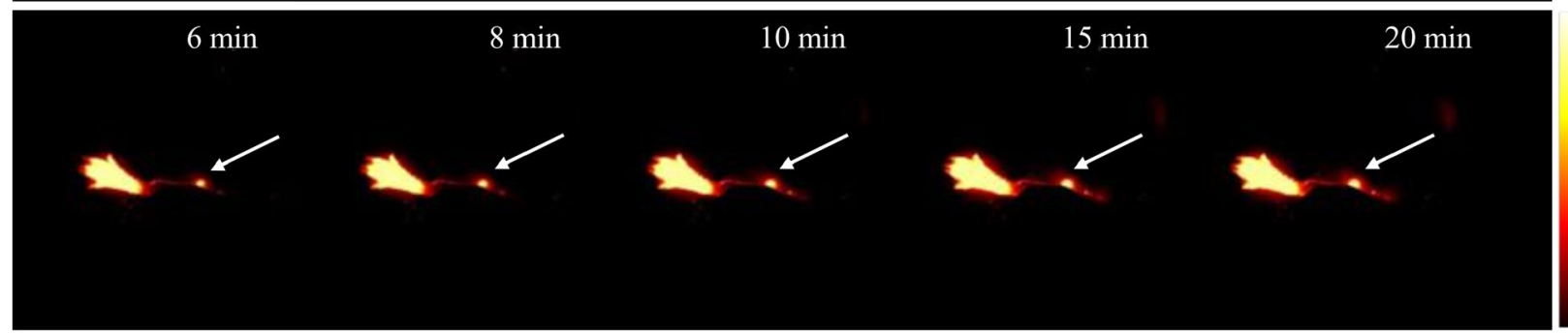

Fig. 3 Fluorescence imaging of mouse vasculature in the NIR-II window (excitation = 915 nm, $1250 \mathrm{~nm}$ long-pass filter, 100 ms expose time). a NIR-II imaging of hind limb vasculature. $\mathbf{b}$ NIR-II imaging of abdominal vessels. c NIR-II imaging of mouse brain through intact scalp and skull vasculature. $\mathbf{d}$ Lymph node imaging after injection of NIC-ER in footpad, white arrows indicate the popliteal lymph node

as a promising small-molecule organic dye with better imaging clarity and quality.

Inspired by the above excellent results, we further investigated the potential of NIC-ER for the precise detection of the tumor. The 4T1 tumor-bearing nude mice were injected with NIC-ER via tail vein. In vivo NIR-II imaging was performed at $1 \mathrm{~min}, 1,2,6,12$, and $24 \mathrm{~h}$ post-injection. The tumor-feeding blood vessels were unambiguously observed from the imaging data at $1 \mathrm{~min}$. During the test time, the signal intensity in the tumor site gradually enhanced. The tumor could be clearly visualized from the surrounding background during 6-24 h post-injection (Fig. 4a). To further track the biodistribution of NIC-ER, the mice were sacrificed at $24 \mathrm{~h}$ post injection and their main organs were harvested for ex vivo imaging (Additional file 1: Figure S8). Strong NIR-II fluorescence signals at the liver, kidney and gut were observed, which suggested NIC-ER could be excreted from the body through hepatobiliary and renal system. The high tumor targeting efficacy of NICER can be ascribed to the albumin-hitchhiking capability of NIC-ER in vivo. After IV injection, NIC-ER was spontaneously located into the pocket of albumin. Thus, the complex can take advantage of endogenous albumin transport pathways for tumor accumulation because tumor microenvironment overexpresses proteins that bind albumin, such as gp60 receptor and SPARC. Moreover, albumin is a macromolecule, and as such the EPR effect may also contribute to tumor uptake. Additionally, slow release of the NIC-ER from albumin over time would allow continual tumor uptake via RGD-mediated endocytosis. Collectively, the effective tumor uptake of NIC-ER can be ascribed to the result of a combination of RGD-mediated endocytosis, EPR effect and endogenous albumin transport pathways. This tumor targeting pattern in other tEB conjugates have been carefully investigated by Chen's group. [32-36]. 

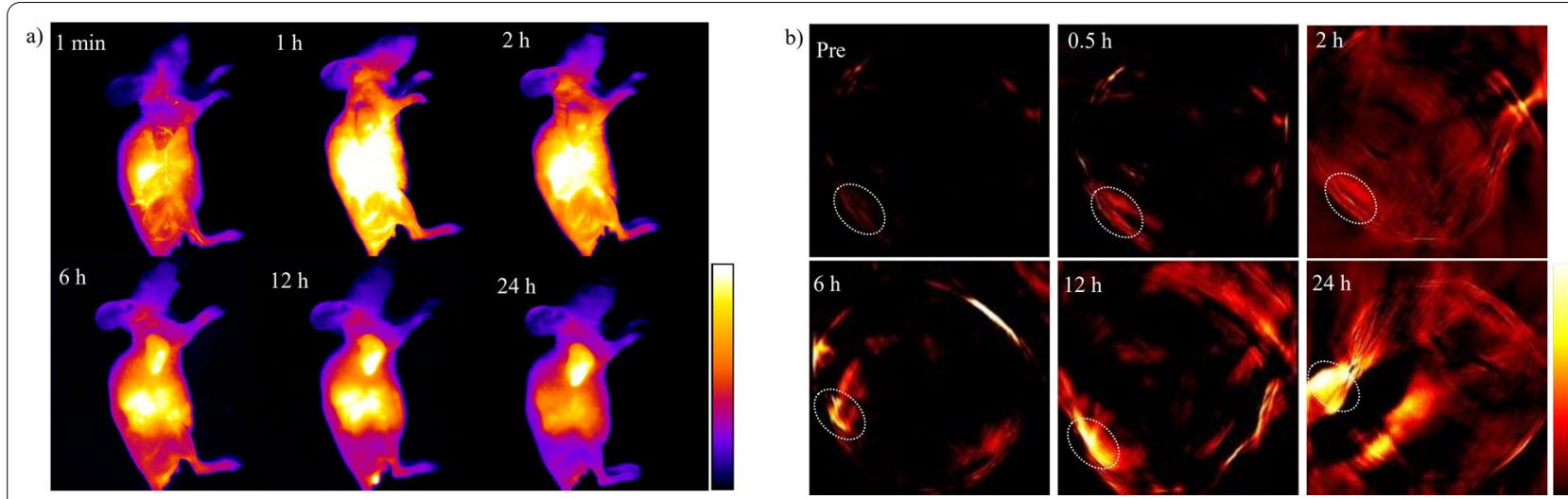

Fig. 4 a The in vivo NIR-II images of the $4 \mathrm{~T} 1$ tumor at different time points (1 min-24 h) after a tail vein injection of NIC-ER. b The in vivo PA images of the 4T1 tumor at different time points (pre-24 h) after a tail vein injection of NIC-ER, the dashed ellipses indicate the tumor

We also performed block study in in vivo NIR II imaging. Given the rapid clearance of RGD and slow pharmacokinetics of NIC-ER, the conjugates of maleimide-modified tEB and $c$ (RGDfc) (termed as ER) was used in vivo to block the $\alpha_{\mathrm{v}} \beta_{3}$ integrin receptor. We can still observe gradually enhanced NIR II signal in tumor site, although the intensity was significantly lower than that in only NIC-ER group at all time points (Additional file 1: Figure S9). This result may indicate that the albumin binding NIC-ER can still accumulate in tumor site by EPR effect or endogenous albumin transport pathways although the $\alpha_{\mathrm{v}} \beta_{3}$ integrin receptor was blocked. Thus, the high tumor targeting efficacy of NIC-ER can largely be ascribed to the combination effect of RGD-mediated endocytosis, EPR effect and endogenous albumin transport pathways.

As mentioned above, NIC-ER exhibited strong absorption in the NIR region, making it have great potential as a PA imaging agent. Thus, the in vivo PA imaging was performed on a subcutaneous $4 \mathrm{~T} 1$ breast cancer tumor-bearing mouse model. The PA tomography system recorded the PA images of tumor cross-sections at different time intervals. Figure $4 \mathrm{~b}$ shows that a very weak PA signal can be observed under the excitation at 910 $\mathrm{nm}$ before intravenous injection of the NIC-ER, which may ascribe to the endogenous hemoglobin contrast in blood vessels. The PA signal of tumor tissue strengthened with the increase of time interval after the injection of NIC-ER, which is consistent with the in vivo NIR-II FL imaging behavior. These excellent imaging results demonstrate that NIC-ER offers an outstanding promise as a NIR-II and PA probe for the margin assessment of tissue localization and intraoperative surgery.

In cancer treatment, efficient therapy is always a challenging task [49]. Recently, photothermal therapy (PTT) has been proven to be a powerful therapeutic modality for cancer [50-53]. Thus, we investigated the possibility of NIC-ER for in vivo photothermal therapy. The temperature of NIC-ER in PBS or HSA solution was first monitored with the irradiation of the $808 \mathrm{~nm}$ or $915 \mathrm{~nm}$ laser at $0.5 \mathrm{w} / \mathrm{cm}^{2}$. Figure $\mathrm{S} 4$ shows that the temperature of NICER/HSA increased quickly in the first $3 \mathrm{~min}$, while the rate decreased and reached a plateau within $5 \mathrm{~min}$. The maximum temperature $\left(\mathrm{T}_{\max }\right)$ of NIC-ER/HSA reached $59{ }^{\circ} \mathrm{C}$ and was maintained at least for $10 \mathrm{~min}$. However, free NIC-ER showed a slower temperature increment rate and lower maximum temperature $\left(\mathrm{T}_{\max }\right)$ when irradiated for the same time. Notably, the temperature of free NICER was slightly decreased with the irradiation time above 13 min, which supports the previous observation of its slight instability under long-time light irradiation. Therefore, the stability and photothermal conversion ability of NIC-ER were improved after being bound to HSA, which would be helpful for cancer phototherapy. In order to visually evaluate the in vitro therapeutic effect of NIC-ER, the cells were stained with calcein-AM and propidium iodide (PI) to identify live and dead/late apoptotic cells, respectively. As shown in Additional file 1: Figure S10, cells all displayed green fluorescence in NIC-ER group, which suggested that NIC-ER alone cannot kill cells. On the contrary, most of cells death and exhibiting intense homogeneous red fluorescence in NIC-ER+Llaser group.

The mice bearing 4T1 subcutaneous tumor were divided randomly into four groups (three mice in each group), which were named "saline", "saline+laser", "NICER", and "NIC-ER + laser", respectively. For the PTT treatment group, the tumors of mice were continuously irradiated with a $915 \mathrm{~nm}$ laser for $5 \mathrm{~min}$ at $12 \mathrm{~h}$ after intravenous injection of NIC-ER. The in vivo antitumor efficacies of the treatments mentioned above were examined by monitoring the tumor volumes for 14 days (the treatments were performed on day 0 ). The tumors treated 


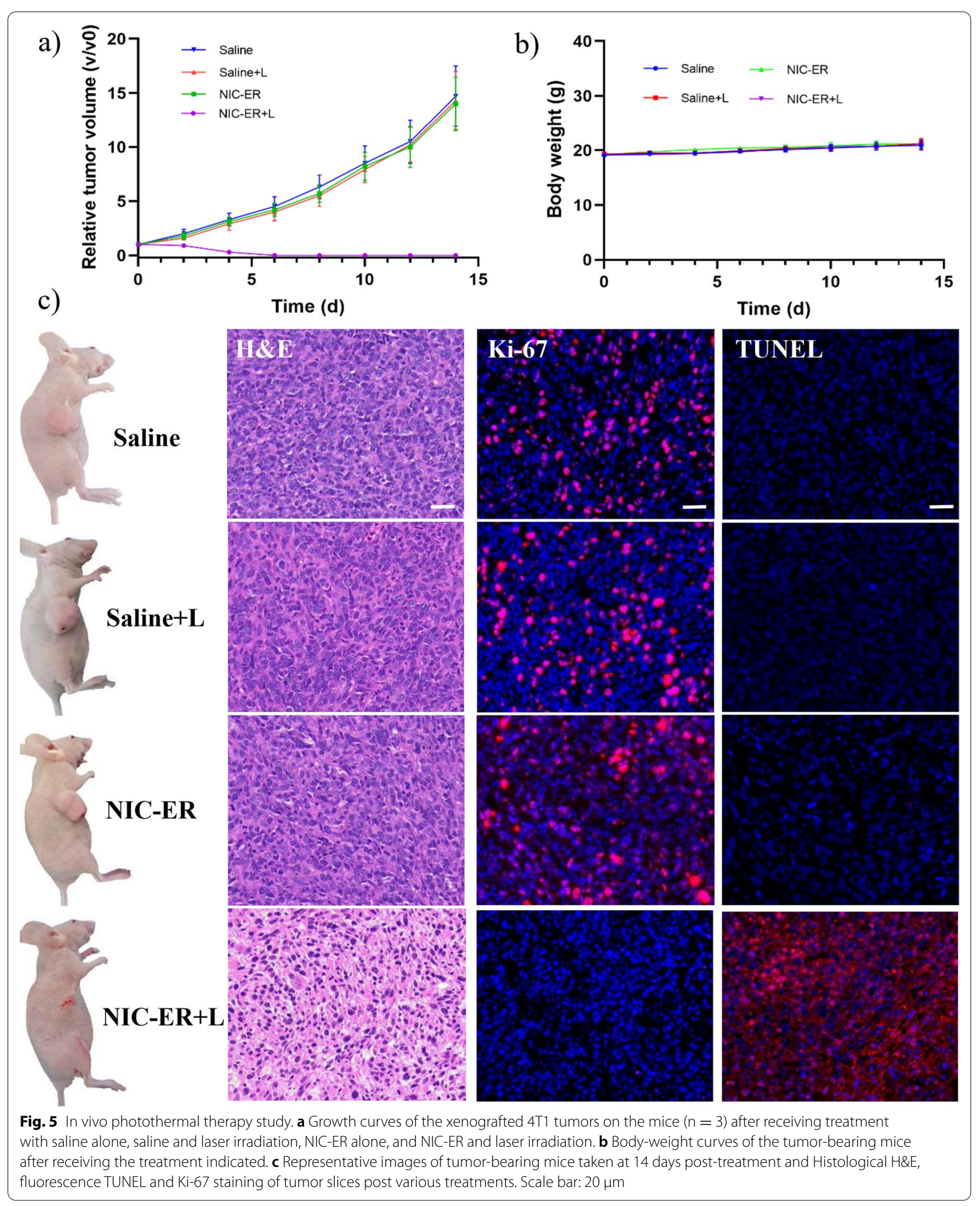


with saline, laser, and NIC-ER without laser irradiation grew rapidly, indicating that the $4 \mathrm{~T} 1$ tumor growth werenot affected by saline, NIC-ER, or laser alone irradiation. In contrast, the "NIC-ER + laser" treatment via PTT exhibits an excellent antitumor efficacy, the growth of the 4T1 tumor was completely inhibited and did not recur during the experiment time (Fig. 5a). Notably, the excellent photothermal activity and the prominent tumor uptake ability of NIC-ER affords such highly effective tumor growth suppression. In addition, there is no significant body weight change in mice in these four groups, suggesting that photothermal treatment was rationally well-tolerated (Fig. 5b).

Furthermore, we studied the therapeutic effect of these four groups at the microscopic level (Fig. 5c). In this experiment, the mice in all four groups were sacrificed, and the tumors were excised for hematoxylin and eosin (H\&E) staining and immune-histochemical analyses, including terminal deoxynucleotidyl transferase dUTP nick end labeling (TUNEL) and transcription factors Ki-67 (Ki-67). Figure 5c shows that tumors in control groups (saline, saline + laser, and NIC-ER) had tightly packed cancer cells with normal morphology, and no necrosis or obvious apoptosis was observed. In contrast, the obvious nuclear reduction was observed in the "NIC-ER + laser" treatment group. The TUNEL staining results showed that the apoptotic cells of tumor tissues had significantly increased in "NIC-ER + laser" treatment group, but not in the other three groups. Ki-67 staining results indicated that the proliferation capacity of tumor tissue was obviously reduced in "NIC-ER + laser" treatment group as compared to those in the other three groups. Both TUNEL and Ki-67 staining confirm that "NIC-ER + laser" treatment is the most efficacious in inducing apoptosis and suppressing the proliferation capacity of tumor cells. These results proved the great potential of NIC-ER as a highly effective therapeutic agent.

\section{Conclusions}

In summary, this study provides a delicate molecular design strategy for developing superior NIR-II cyanine dye with sufficient brightness, large Stokes-shift, and high photostability. Compared to the symmetrical cyanine dye ICG, NIC has a nonsymmetrical structure by replacing only indoline donors with a fully conjugated benz $[\mathrm{c}, \mathrm{d}]$ indole donor. This minor structure alternation affords NIC with NIR-II emission and high extinction coefficient. NIC-ER with endogenous albumin-hitchhiking capability was constructed to further enhance its in vivo fluorescence brightness. In the presence of albumin, NIC-ER was spontaneously located into the pocket of albumin, and a brilliant $\sim 89$-fold increase in fluorescence was observed. NIC-ER in HSA exhibits bright NIR-II emission with high photostability and significant Stokes shift $(>110 \mathrm{~nm})$ due to the high molar absorptivity and moderate quantum yield. NIC-ER exhibits NIR-II/PA bimodal imaging capabilities and favorable photothermal effect. Thus, noninvasive NIRII imaging of small blood vessels at limb, abdomen, and cerebral regions was achieved with a high signalto-background ratio and deep penetration. NIC-ER has also been used for targeted NIR-II fluorescence and NIR-I PA imaging of subcutaneous tumors. Moreover, NIC-ER achieved efficient photothermal elimination of tumors without recurrence due to its excellent photothermal conversion capability and tumor-targeting ability. The above results proved that NIC-ER has great potential in clinical applications for tumor imaging and photothermal therapy. Overall, our strategy may open a new avenue for designing and constructing high-performance NIR-II fluorophores.

\section{Supplementary Information}

The online version contains supplementary material available at https://doi. org/10.1186/s12951-021-01075-0.

Additional file 1: Figure S1. Cell viability of Hela cells after incubated with NIC-ER with various concentrations for $48 \mathrm{~h}$. Figure S2. Absorbance intensity change of NIC-ER in HSA/PBS/Blood and ICG-COOH in PBS after repeated laser irradiation. NIR-II fluorescence image of NIC-ER in HSA or Blood. Figure S3. Photo of ICG-COOH (left) and NIC (right) in DMSO. Figure S4. Temperature curves of NIC-ER in HSA or in PBS and HSA alone under NIR laser irradiation. Figure S5. NIR-II imaging of hind limb vasculature with the vessel FWHM width (white line) analysis. Scale bar: $5 \mathrm{~mm}$. Figure S6. NIR-II imaging of cerebral vasculature with the vessel FWHM width (white line) analysis. Scale bar: $5 \mathrm{~mm}$. Figure S7. Histological H\&E staining for main organs (heart, liver, spleen, lung and kidney) of the mice intravenously administrated with PBS and NIC-ER (10 times than the imaging doses, $5 \mathrm{mg}$ of NIC-ER per kg mouse) for 7 days. Scale bar: $50 \mu \mathrm{m}$. Figure S8. Ex vivo images of main organs of NIC-ER treated mice. Figure S9. The in vivo NIR-II images of the 4T1 tumor at different time points (1-24 h) after CO-injection of NIC-ER and ER (the conjugates of EB and CRGD, $0.4 \mathrm{mg} /$ mouse). Figure S10. Calcein-AM/propidium iodide (PI) staining of $4 \mathrm{~T} 1$ cells pretreated with NIC-ER with or without laser for $5 \mathrm{~min}$ at $0.5 \mathrm{~W} / \mathrm{cm}^{2}$. Table $\mathbf{S 1}$. Overview of the names and chemical structures of key compounds. Table S2. The absolute quantum yield of NIC-ER in DMSO, PBS and HSA (3 mg/mL). Scheme S1. The synthetic route of NIC-

ER. Figure S11. 1H NMR spectroscopy of compound of NIC. Figure S12. 13C NMR spectroscopy of compound of NIC. Figure S13. MS spectroscopy of NIC. Figure S14. MALDI-TOF-MS measurement of NIC-MLEB.

Figure S15. MALDI-TOF-MS measurement of NIC-ER.

\section{Acknowledgements}

The authors thank Dr. Xin Yan from Sun Yat-sen University for valuable suggestions and support about the theoretical calculation and docking modeling studies.

\section{Author's contributions}

$J W, P J, W Y$ and PX designed and conceptualized this study. PX, LH, CY, FK and $M Z$. performed the in vitro and in vivo experiments. PX, LH,WY and JW drafted the manuscript. All authors read and approved the final manuscript. 


\section{Funding}

This work was supported by National Natural Science Foundation of China (No. 81701754, 81871379, 81801730, 81971646, 81602846), China Postdoctoral Science Foundation (2020M673660), Major Research Plan of National Natural Science Foundation of China (91959208), Taishan Scholar Project of Shandong Province (tsqn201812159) and The Key Research and Development Program of Jining Science and Technology (2019SMNS012).

\section{Availability of data and materials}

All data generated or analysed during this study are included in this published article.

\section{Declarations}

\section{Ethics approval and consent to participate}

All of the cell and animal studies were approved by the Institutional Animal Care and Use Committee of Jining First People's Hospital (JNRM 2020-023).

\section{Consent for publication}

All authors agree to be published.

\section{Competing interests}

The authors declare that they have no conflict of interest.

\section{Author details}

${ }^{1}$ Department of Nuclear Medicine, Xijing Hospital, Fourth Military Medical University, \#127 West Changle Road, Shanxi 710032 Xi'an, People's Republic of China. ${ }^{2}$ Institute of Clinical Pharmacy and Pharmacology, Jining First People's Hospital, Jining Medical University, Jining 272000, People's Republic of China. ${ }^{3}$ Departments of Radiology, The Second Xiangya Hospital, Central South University, Changsha, Hunan 410011, People's Republic of China.

\section{Received: 25 May 2021 Accepted: 8 October 2021}

\section{Published online: 24 October 2021}

\section{References}

1. Cao J, Zhu B, Zheng K, He S, Meng L, Song J, et al. Recent progress in NIR-II contrast agent for biological imaging. Front Bioeng Biotechnol. 2019;7:487-508.

2. Xu W, Wang D, Tang BZ. NIR-II AlEgens: a win-win integration towards bioapplications. Angew Chem Int Ed Engl. 2020;59:2-14.

3. Chen G, Cao Y, Tang Y, Yang X, Liu Y, Huang D, et al. Advanced near-infrared light for monitoring and modulating the spatiotemporal dynamics of cell functions in living systems. Adv Sci (Weinh). 2020;7(8):1903783-810.

4. He S, Song J, Qu J, Cheng Z. Crucial breakthrough of second near-infrared biological window fluorophores: design and synthesis toward multimodal imaging and theranostics. Chem Soc Rev. 2018;47(12):4258-78.

5. Chen J, Kong Y, Wang W, Fang H, Wo Y, Zhou D, et al. Direct water-phase synthesis of lead sulfide quantum dots encapsulated by beta-lactoglobulin for in vivo second near infrared window imaging with reduced toxicity. Chem Commun (Camb). 2016:52(21):4025-8.

6. Naczynski DJ, Tan MC, Zevon M, Wall B, Kohl J, Kulesa A, et al. Rare-earthdoped biological composites as in vivo shortwave infrared reporters. Nat Commun. 2013;4:2199-209.

7. Perrault SD, Walkey C, Jennings T, Fischer HC, Chan WC. Mediating tumor targeting efficiency of nanoparticles through design. Nano Lett. 2009;9(5):1909-15.

8. Zhu S, Yung BC, Chandra S, Niu G, Antaris AL, Chen X. Near-infrared-II (NIR-II) bioimaging via off-peak NIR-I fluorescence emission. Theranostics. 2018:8(15):4141-51

9. Antaris AL, Chen H, Cheng K, Sun Y, Hong G, Qu C, et al. A small-molecule dye for NIR-II imaging. Nat Mater. 2016;15(2):235-42.

10. Shou K, Qu C, Sun Y, Chen H, Chen S, Zhang L, et al. Multifunctional biomedical imaging in physiological and pathological conditions using a NIR-II probe. Adv Funct Mater. 2017;27(23).

11. Sun Y, Qu C, Chen H, He M, Tang C, Shou K, et al. Novel benzo-bis(1,2,5thiadiazole) fluorophores for in vivo NIR-II imaging of cancer. Chem Sci. 2016;7(9):6203-7.
12. Zhang XD, Wang $H$, Antaris $A L$, Li L, Diao S, Ma R, et al. Traumatic brain injury imaging in the second near-infrared window with a molecular fluorophore. Adv Mater. 2016;28(32):6872-9.

13. Xu P, Kang F, Yang W, Zhang M, Dang R, Jiang P, et al. Molecular engineering of a high quantum yield NIR-II molecular fluorophore with aggregation-induced emission (AIE) characteristics for in vivo imaging. Nanoscale. 2020;12(8):5084-90.

14. Kenry, Duan Y, Liu B. Recent advances of optical imaging in the second near-infrared window. Adv Mater. 2018;30(47):e1802394.

15. Laissue PP, Alghamdi RA, Tomancak P, Reynaud EG, Shroff H. Assessing phototoxicity in live fluorescence imaging. Nat Methods. 2017;14(7):657-61.

16. Qi J, Duan X, Liu W, Li Y, Cai Y, Lam JWY, et al. Dragonfly-shaped nearinfrared AIEgen with optimal fluorescence brightness for precise imageguided cancer surgery. Biomaterials. 2020;248:120036.

17. Carr JA, Franke D, Caram JR, Perkinson CF, Saif M, Askoxylakis V, et al. Shortwave infrared fluorescence imaging with the clinically approved near-infrared dye indocyanine green. Proc Natl Acad Sci USA. 2018;115(17):4465-70.

18. Sun W, Guo S, Hu C, Fan J, Peng X. Recent development of chemosensors based on cyanine platforms. Chem Rev. 2016;116(14):7768-817.

19. Kaplan-Marans E, Fulla J, Tomer N, Bilal K, Palese M. Indocyanine green (ICG) in urologic surgery. Urology. 2019;132:10-7.

20. Alander JT, Kaartinen I, Laakso A, Patila T, Spillmann T, Tuchin W, et al. A review of indocyanine green fluorescent imaging in surgery. Int J Biomed Imaging. 2012;2012:940585

21. Beckford G, Owens E, Henary M, Patonay G. The solvatochromic effects of side chain substitution on the binding interaction of novel tricarbocyanine dyes with human serum albumin. Talanta. 2012;92:45-52.

22. Li B, Lu L, Zhao M, Lei Z, Zhang F. An efficient 1064 nm NIR-II excitation fluorescent molecular dye for deep-tissue high-resolution dynamic bioimaging. Angew Chem Int Ed Engl. 2018;57(25):7483-7.

23. Tian R, Ma H, Zhu S, Lau J, Ma R, Liu Y, et al. Multiplexed NIR-II probes for lymph node-invaded cancer detection and imaging-guided surgery. Adv Mater. 2020;32(11):e1907365.

24. Zhu S, Hu Z, Tian R, Yung BC, Yang Q, Zhao S, et al. Repurposing cyanine NIR-I dyes accelerates clinical translation of near-infrared-II (NIR-II) Bioimaging Adv Mater. 2018:e1802546.

25. Zhu S, Tian R, Antaris AL, Chen X, Dai H. Near-infrared-II molecular dyes for cancer imaging and surgery. Adv Mater. 2019:31(24):e1900321.

26. Gayton J, Autry SA, Meador W, Parkin SR, Hill GA Jr, Hammer NI, et al. Indolizine-cyanine dyes: near infrared emissive cyanine dyes with increased stokes shifts. J Org Chem. 2019;84(2):687-97.

27. Upadhyayula S, Nunez V, Espinoza EM, Larsen JM, Bao D, Shi D, et al. Photoinduced dynamics of a cyanine dye: parallel pathways of non-radiative deactivation involving multiple excited-state twisted transients. Chem Sci. 2015;6(4):2237-51.

28. Tian R, Zeng Q, Zhu S, Lau J, Chandra S, Ertsey R, et al. Albumin-chaperoned cyanine dye yields superbright NIR-II fluorophore with enhanced pharmacokinetics. Sci Adv. 2019;5(9):eaaw0672.

29. Antaris $A L$, Chen $H$, Diao S, Ma Z, Zhang Z, Zhu S, et al. A high quantum yield molecule-protein complex fluorophore for near-infrared II imaging. Nat Commun. 2017:8:15269.

30. Xie L, Wang G, Zhou H, Zhang F, Guo Z, Liu C, et al. Functional long circulating single walled carbon nanotubes for fluorescent/photoacoustic imaging-guided enhanced phototherapy. Biomaterials. 2016;103:219-28.

31. Zhang L, Gao S, Zhang F, Yang K, Ma Q, Zhu L. Activatable hyaluronic acid nanoparticle as a theranostic agent for optical/photoacoustic imageguided photothermal therapy. ACS Nano. 2014;8(12):12250-8.

32. Wang $Y$, Lang L, Huang $P$, Wang $Z$, Jacobson $O$, Kiesewetter DO, et al. In vivo albumin labeling and lymphatic imaging. Proc Natl Acad Sci USA. 2015;112(1):208-13

33. Tian R, Zhu S, Zeng Q, Lang L, Ma Y, Kiesewetter DO, et al. An albumin sandwich enhances in vivo circulation and stability of metabolically labile peptides. Bioconjug Chem. 2019;30(6):1711-23.

34. Chen H, Jacobson O, Niu G, Weiss ID, Kiesewetter DO, Liu Y, et al. Novel "Add-On" molecule based on evans blue confers superior pharmacokinetics and transforms drugs to theranostic agents. J Nucl Med. 2017:58(4):590-7.

35. Ehlerding EB, Lan X, Cai W. "Albumin Hitchhiking" with an evans blue analog for cancer theranostics. Theranostics. 2018;8(3):812-4. 
36. Um W, Park J, Youn A, Cho H, Lim S, Lee JW, et al. A comparative study on albumin-binding molecules for targeted tumor delivery through covalent and noncovalent approach. Bioconjug Chem. 2019;30(12):3107-18.

37. Wang Z, Tian R, Niu G, Ma Y, Lang L, Szajek LP, et al. Single low-dose injection of evans blue modified PSMA-617 radioligand therapy eliminates prostate-specific membrane antigen positive tumors. Bioconjug Chem. 2018;29(9):3213-21.

38. Zhang J, Lang L, Zhu Z, Li F, Niu G, Chen X. Clinical translation of an albumin-binding PET radiotracer 68Ga-NEB. J Nucl Med. 2015;56(10):1609-14.

39. Lei Z, Zhang F. Molecular engineering of NIR-II fluorophores for improved biomedical detection. Angew Chem Int Ed Engl. 2021;60(30):16294-308.

40. Du Y, Liu X, Zhu S. Near-infrared-II cyanine/polymethine dyes, current state and perspective. Front Chem. 2021;9:718709-14.

41. Liu R, Tang J, Xu Y, Zhou Y, Dai Z. Nano-sized indocyanine green $J$-aggregate as a one-component theranostic agent. Nanotheranostics. 2017;1(4):430-9.

42. Yarmoluk SM, Lukashov SS, Ogul'Chansky TY, Losytskyy MY, Kornyushyna OS. Interaction of cyanine dyes with nucleic acids. XXI. Arguments for half-intercalation model of interaction. Biopolymers. 2001;62(4):219-27.

43. Zhang Z, Kao J, D'Avignon A, Achilefu S. Understanding dichromic fluorescence manifested in certain ICG analogs. Pure Appl Chem. 2010;82(1):307-11.

44. Brooker LGS, Keyes GH, Williams WW. Color and Constitution. V.1 The Absorption of unsymmetrical cyanines. Resonance as a basis for a classification of dyes. 1942;64: 199-210.

45. Cosco ED, Caram JR, Bruns OT, Franke D, Day RA, Farr EP, et al. Flavylium polymethine fluorophores for near- and shortwave infrared imaging. Angew Chem Int Ed Engl. 2017;56(42):13126-9

46. Hong G, Zou Y, Antaris AL, Diao S, Wu D, Cheng K, et al. Ultrafast fluorescence imaging in vivo with conjugated polymer fluorophores in the second near-infrared window. Nat Commun. 2014:5:4206.
47. Kong Y, Chen J, Fang H, Heath G, Wo Y, Wang W, et al. Highly fluorescent ribonuclease-a-encapsulated lead sulfide quantum dots for ultrasensitive fluorescence in vivo imaging in the second near-infrared window. Chem Mater. 2016;28(9):3041-50.

48. van Beurden F, van Willigen DM, Vojnovic B, van Oosterom MN, Brouwer $\mathrm{OR}$, der Poel HGV, et al. Multi-wavelength fluorescence in imageguided surgery, clinical feasibility and future perspectives. Mol Imaging. 2020;19:1536012120962333.

49. Guo B, Sheng Z, Hu D, Liu C, Zheng H, Liu B. Through scalp and skull NIR-II photothermal therapy of deep orthotopic brain tumors with precise photoacoustic imaging guidance. Adv Mater. 2018;30(35):e1802591.

50. Jung HS, Verwilst P, Sharma A, Shin J, Sessler JL, Kim JS. Organic moleculebased photothermal agents: an expanding photothermal therapy universe. Chem Soc Rev. 2018;47(7):2280-97.

51. Li S, Deng Q, Zhang Y, Li X, Wen G, Cui X, et al. Rational design of conjugated small molecules for superior photothermal theranostics in the NIR-II biowindow. Adv Mater. 2020;32(33):e2001146.

52. Liu Y, Bhattarai P, Dai Z, Chen X. Photothermal therapy and photoacoustic imaging via nanotheranostics in fighting cancer. Chem Soc Rev. 2019;48(7):2053-108

53. Zhi D, Yang T, O'Hagan J, Zhang S, Donnelly RF. Photothermal therapy. J Control Release. 2020;325:52-71.

\section{Publisher's Note}

Springer Nature remains neutral with regard to jurisdictional claims in published maps and institutional affiliations.
Ready to submit your research? Choose BMC and benefit from:

- fast, convenient online submission

- thorough peer review by experienced researchers in your field

- rapid publication on acceptance

- support for research data, including large and complex data types

- gold Open Access which fosters wider collaboration and increased citations

- maximum visibility for your research: over 100M website views per year

At BMC, research is always in progress.

Learn more biomedcentral.com/submissions 